\title{
TIME DEPENDANT QUANTUM EFFICIENCY AND DARK CURRENT MEASUREMENTS IN AN RF PHOTOCATHODE INJECTOR WITH A HIGH QUANTUM EFFICIENCY CATHODE*
}

\author{
R.P. Fliller III ${ }^{\#}$, H. Edwards, Fermilab, Batavia, IL 60510 \\ W. Hartung, NSCL, Michigan State University, East Lansing, MI 48824
}

\begin{abstract}
Studies of photo-emission and field emission behavior in an RF gun have been carried out. Unexpected phenomena were observed. In situ changes in the cathode's quantum efficiency and dark current with time were seen during operation of the photo-injector. These changes were correlated with the magnetostatic field at the cathode. In addition, multipacting has been observed under certain conditions. Recent measurements indicate a correlation between multipacting and anomalous photoand field emission behavior.
\end{abstract}

\section{INTRODUCTION}

The Fermilab NICADD Photo-Injector Laboratory (FNPL) is an $18 \mathrm{MeV}$ electron linac operating in partnership with Northern Illinois University. The injector consists of a 1.625-cell RF electron gun with a high quantum efficiency $\mathrm{Cs}_{2} \mathrm{Te}$ photocathode $[1,2]$. The system for preparing and transferring the photo-cathodes was developed by INFN Milano; a similar system is used at the TeSLA Test Facility at DESY-Hamburg [3]. A pulsed ultra-violet (UV) laser is used to produce photoelectrons [4]. Further acceleration of the beam is provided by a 9-cell superconducting cavity [5].

Previous studies have shown an anomalous time dependence in the quantum efficiency of the photocathode and the field emission ("dark current") of the RF gun correlated with the magnetostatic field configuration in the gun [6]. We observed that the solenoid settings for normal operation were correlated with an asymptotic increase in both the quantum efficiency and dark current with time. The quantum efficiency and dark current decreased asymptotically for two other combinations of solenoid settings.

Multipacting is observed in the RF gun with the normal solenoid settings. Recent measurements indicate a correlation between multipacting, anomalous photoemission behavior, and anomalous field emission behavior [7].

In this paper, we will parameterize the time-dependent dark current using the Fowler-Nordheim parameters [8]. We will review some of the evidence linking multipacting to the photo-emission and field emission anomalies. We will also show that, for a given RF field, the multipacting behavior is determined predominantly by the magnetostatic field at the cathode.

\footnotetext{
* This work was supported by Universities Research Association Inc. under contract DE-AC02-76CH00300 with the U.S. DOE and by NICADD.

\# Email: fliller@fnal.gov
}

\section{PHOTOINJECTOR DESCRIPTION}

The RF gun is a $1.3 \mathrm{GHz} 1.625$-cell copper cavity that has been in use since March 1999. The maximum RF amplitude corresponds to a peak surface electric field of $35 \mathrm{MV} / \mathrm{m}$ at the cathode and a beam energy at the exit of the gun of $4.5 \mathrm{MeV}$. For the present measurements, the RF pulse duration was between 20 and $100 \mu$ s and the repetition rate was $1 \mathrm{~Hz}$.

The gun is surrounded by three focusing solenoids, referred to as "bucking," "primary," and "secondary." The primary solenoid encircles the half-cell of the gun, while the secondary solenoid is located $240 \mathrm{~mm}$ from the cathode. The bucking solenoid is located behind the gun and is used to make $B_{z}$ approximately zero at the cathode under normal "round beam" conditions. To make "flat beams" with asymmetric emittances, the bucking solenoid is not used and $B_{z}=800$ Gauss at the cathode [9].

The photo-injector uses UV pulses of duration $10 \mathrm{ps}$ FWHM from the laser directed onto the $\mathrm{Cs}_{2} \mathrm{Te}$ cathode inside the RF gun. The UV energy per pulse and charge per photo-electron bunch were measured with a photodiode and Integrating Current Transformer (ICT, located $0.38 \mathrm{~m}$ downstream of the gun), respectively, allowing us to infer the quantum efficiency. Thus the quantum efficiency includes not only the intrinsic efficiency of the cathode, but also takes into account the transport of the photo-electrons from the cathode into the beam tube.

The dark current was measured with a Faraday cup inserted into a diagnostic port $0.07 \mathrm{~m}$ after the ICT. As with the quantum efficiency, the dark current also includes the transport from the cathode.

\section{ANOMALOUS BEHAVIOR}

As was noted in the Reference 6, it was observed that the quantum efficiency and dark current asymptotically increased with time with round beam solenoid settings. With flat beam settings or solenoids off, the quantum efficiency and dark current asymptotically decreased. These observations were initially made with another cathode, but recent measurements on the current cathode show the same behavior, as illustrated in Figure 1.

\section{FIELD EMISSION}

We monitored the dark current as a function of time while running with round beam setting for 45 hours. The dark current versus time is shown in Reference 6, Figure 1a. During this measurement, we paused occasionally to measure the dark current as a function of RF field. 


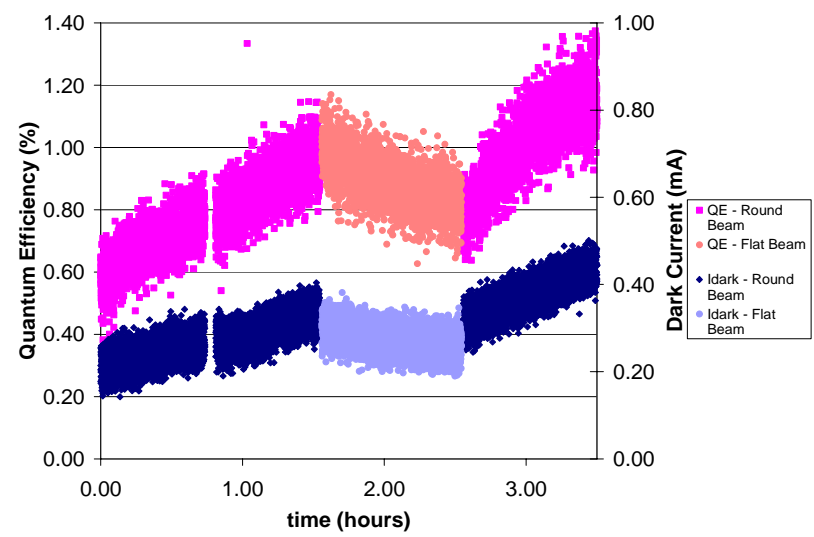

Figure 1: Quantum efficiency and dark current vs. time, measured while switching between round beam and flat beam solenoid settings.

The dark current can be fit to the Fowler-Nordheim formula [8]. Using $4.7 \mathrm{eV}$ for the work function of copper, the Fowler-Nordheim formula for enhanced field emission becomes

$$
I_{\text {dark }}=46.2 \times 10^{-6} A(\beta E)^{2.5} \exp \left(\frac{-6.654 \times 10^{4}}{\beta E}\right)
$$

where $E$ is the cathode electric field in $\mathrm{MV} / \mathrm{m}, \beta$ is the enhancement factor, $A$ is the effective emission area in $\mu \mathrm{m}^{2}$, and $I_{\text {dark }}$ is the dark current in $\mathrm{mA}$.

Using Equation 1, we fitted the repeated measurements of dark current as a function of $E$ and calculated the Fowler-Nordheim parameters as a function of time. Results are shown in Figure 2.

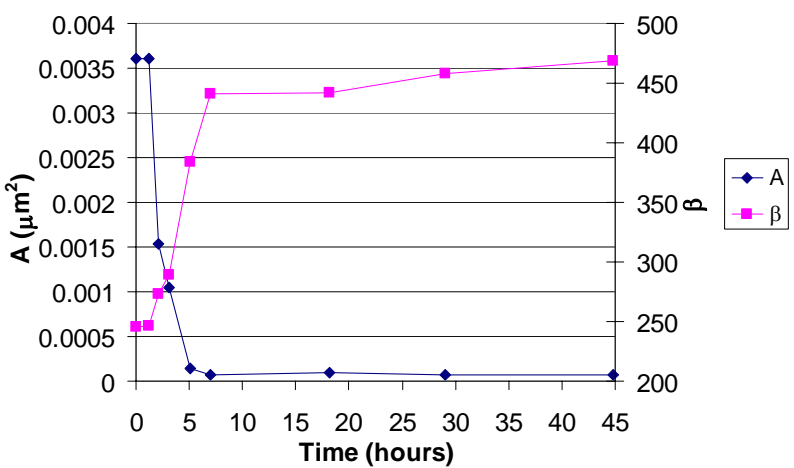

Figure 2: Time dependence of Fowler-Nordheim parameters while running with round beam settings.

The first six hours show the most change, then things change much more slowly. The net effect is that the dark current increases with time with round beam settings.

We also compared the dark current for different solenoid settings. Figure 3 shows the dark current for round beam, flat beam and solenoids off settings before and after 12.5 hours of running with round beam settings. Round beam settings always show more dark current. In the beginning, the flat beam settings show less dark current than solenoids off; at the end the flat beam settings produce more dark current than solenoids off.

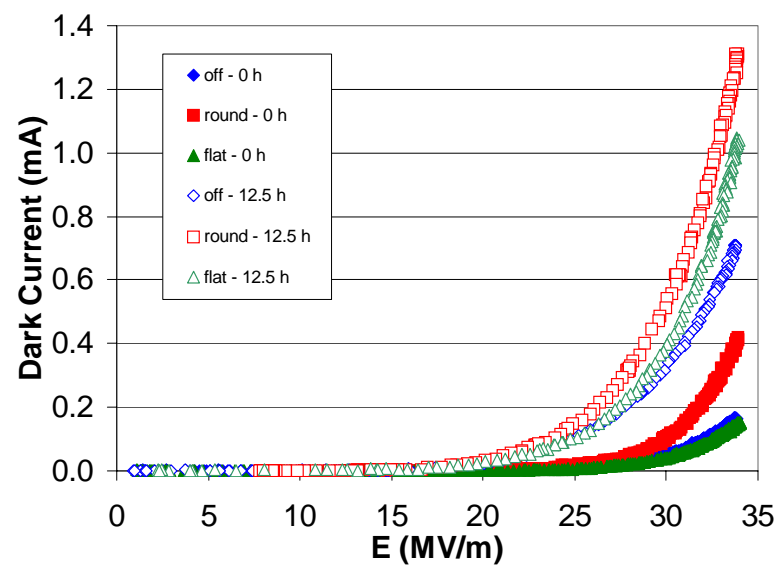

Figure 3: Dark current vs. RF field amplitude with different solenoid settings, before and after running for 12.5 hours with round beam settings.

\section{MULTIPACTING}

With round beam solenoid settings, a pulse of current emerges from the gun after the end of the RF pulse, as the RF field amplitude decreases exponentially. This is shown in Figure 4. A current pulse is sometimes seen at the beginning of the RF pulse also. The current pulses are not observed with flat beam settings or solenoids off.

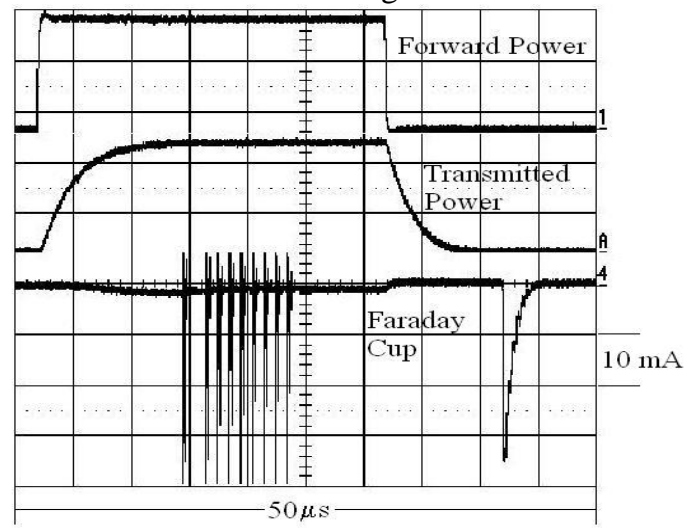

Figure 4: Composite of scope traces showing the forward power (top), transmitted power (middle), and current (bottom) with round beam settings. The multipacting spike appears after the RF is turned off.

We believe that the current pulse is produced by multipacting electrons, with multipacting occurring only with the right combination of magnetostatic and RF fields [7]. ASTRA simulations have confirmed that multipacting can occur in the RF gun [10]. Measurements at the Photo Injector Test Facility at DESY-Zeuthen (PITZ) show similar behavior [10]. We refer to the current pulse as the "multipacting spike."

We cannot say for sure what mechanism is responsible for the correlation between multipacting, photo-emission, and field emission, but our hypothesis is that multipacting in the gun cleans the surface of the photocathode, which allows for an increase in photo-emission and field emission. When multipacting is not present, residual gas 
molecules may stick to the cathode surface and reduce emission. This hypothesis has not been directly tested.

To further investigate the characteristics of the multipacting spike, we measured the delay of the multipacting spike after the RF is turned off while varying the RF amplitude. Results are shown in Figure 5. The data were fit to

$$
t_{\text {delay }}=\tau \ln \left(\frac{E}{E_{m p}}\right)
$$

where $\tau$ is the decay time of the cavity field, and $E_{m p}$ is the field at which the gun multipacts.

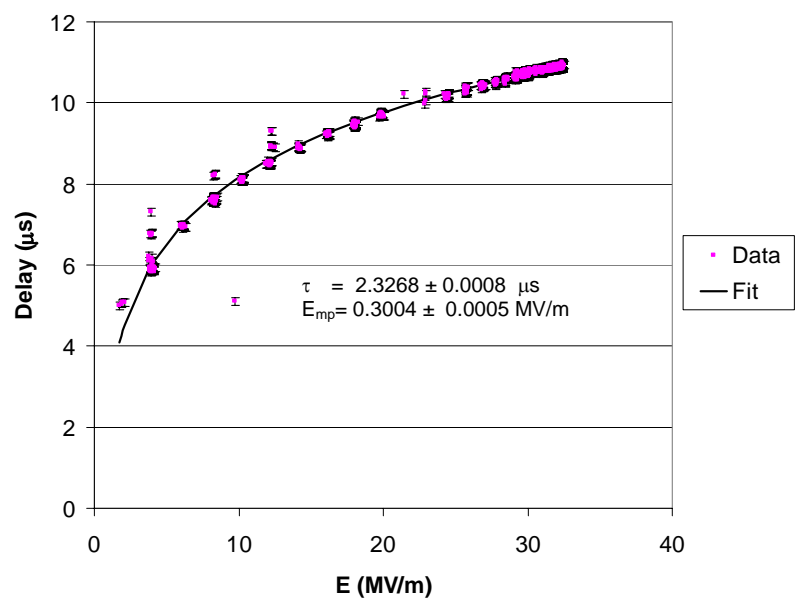

Figure 5: Multipacting spike delay vs. cathode electric field maximum.

The value of $\tau$ is consistent with previous $\mathrm{RF}$ measurements on this gun [11]. The value of $E_{m p}$ is smaller than that observed at PITZ by a factor of 3.2 [10].

Starting from round and flat beam settings, we varied the current in each of the solenoids independently and measured the charge and delay time of the multipacting spike. The current in each solenoid was varied through its entire range.

Figure 6 shows the results for the primary and bucking solenoid variations around round beam settings. The multipacting spike shows similar, but not quite identical behavior when each solenoid is varied. When $B_{\text {cath }}>400$ Gauss, multipacting is no longer present. We also note that there is no symmetry around zero field.

The flat beam case is different. It was possible to cause multipacting if the bucking solenoid was varied to make $B_{\text {cath }}$ less than 100 Gauss. The charge and delay of the multipacting spike does not overlap the traces in Figure 6, but their behavior is very similar. There is no multipacting when $B_{\text {cath }}>100$ Gauss, in contrast to the round beam case. From this we conclude that the axial magnetic and electric field on the cathode are not the only factors in determining whether multipacting is present.

The primary solenoid with no bucking solenoid current could cause multipacting near its upper current limit, which corresponds to $B_{\text {cath }}=1.2 \mathrm{kGauss}$. However, the size of the multipacting spike was so small that no meaningful data was collected.

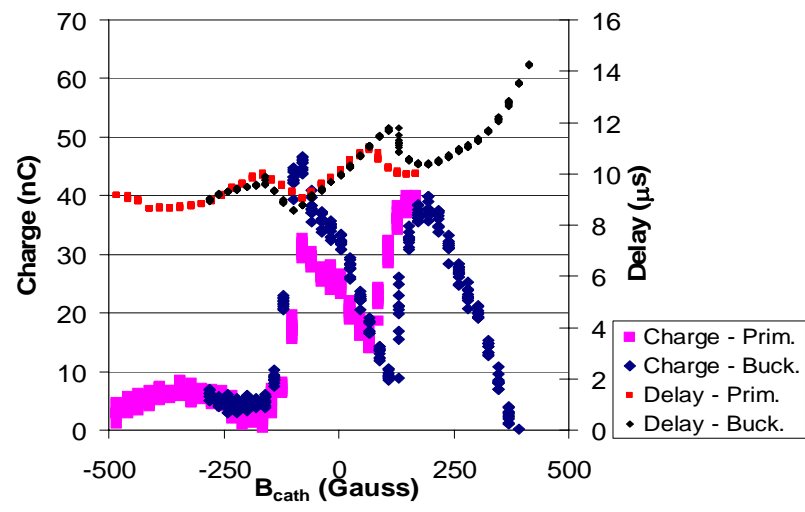

Figure 6: Charge and delay of multipacting spike. The primary and bucking solenoid currents are varied independently around round beam settings.

The secondary solenoid had a negligible effect on the multipacting in both the flat and round beam cases.

\section{CONCLUSIONS}

The quantum efficiency and dark current in the FNPL RF gun displays a time dependant behavior that depends on the magnetostatic field in the gun. The magnetostatic field in the gun causes multipacting in the round beam configuration, which is beneficial to the quantum efficiency and increases field emission. The field emission change is characterized by a time dependence of the Fowler-Nordheim parameters.

We have parameterized the multipacting spike by its dependence on the electric and magnetic field at the cathode. Future studies are planned to simulate the gun under these conditions.

\section{REFERENCES}

[1] E. Colby, in Proceedings of the XIX International Linac Conference, Report ANL-98/28, 1998, p. 758762.

[2] A. Fry et al., Ibid., p. 642-644.

[3] P. Michelato et al., in Proceedings of the Fifth European Particle Accelerator Conference, 1996, p. 1510-1512.

[4] A. R. Fry et al., Nucl. Instrum. Methods Phys. Res. A430, p. 180-188 (1999).

[5] W. Hartung et al., in Proceedings of the 1999 Particle Accelerator Conference, p. 992-994.

[6] W. Hartung et al., in Proceedings of the 2001 Particle Accelerator Conference, p. 2239-2241.

[7] W. Hartung et al., "Photo-Emission and FieldEmission Investigations,” FNPL Advisory Committee Report (October 2002).

[8] J. W. Wang and G. A. Loew, SLAC-PUB-7684 (October 1997).

[9] Y.-E Sun et al., Phys. Rev. ST Accel. Beams 7, 123501 (2004).

[10] J. H. Han, private communication.

[11]J.-P. Carneiro, PhD Thesis, Université Paris XI (2001). 